\title{
The Impact of an Ice Slurry-Induced Gastrointestinal Heat Sink on Gastrointestinal and Rectal Temperatures Following Exercise
}

\author{
Thomas A. Deshayes ${ }^{1,2} \mathbb{D}$, Adrien De La Flore ${ }^{3}$, Jonathan Gosselin ${ }^{1}$, Jeff Beliveau ${ }^{1}$, \\ David Jeker ${ }^{1}$ and Eric D.B. Goulet ${ }^{1,2, *(D)}$ \\ 1 Faculty of Physical Activity Sciences, University of Sherbrooke, Sherbrooke, QC J1K 2R1, Canada \\ 2 Research Centre on Aging, University of Sherbrooke, Sherbrooke, QC J1H 4C4, Canada \\ 3 Institut National des Sciences Appliquées, Université de Toulouse, 31400 Toulouse, France \\ * Correspondence: Eric.Goulet@USherbrooke.ca; Tel.: +1-819-821-8000 (ext. 62728)
}

Received: 30 June 2019; Accepted: 22 August 2019; Published: 27 August 2019

\begin{abstract}
Gastrointestinal temperature (Tgint) measurement with a telemetric pill (TP) is increasingly used in exercise science. Contact of cool water with a TP invalidates Tgint assessment. However, what effect a heat sink created in the proximity of a TP may have on the assessment of Tgint remains unknown. We examined the impact of an ice slurry-induced heat sink on Tgint and rectal temperature (Trec) following exercise. After $20 \mathrm{~min}$ of seating $\left(20-22{ }^{\circ} \mathrm{C}, 25-40 \%\right.$ relative humidity (RH)), 11 men completed two intersperse exercise periods $\left(31-32{ }^{\circ} \mathrm{C}, 35 \% \mathrm{RH}\right)$ at $75-80 \%$ of estimated maximal heart rate until a Trec increase of $1{ }^{\circ} \mathrm{C}$ above baseline level. Following the first exercise period, participants were seated for $45 \mathrm{~min}$ and ingested $7.5 \mathrm{~g} \cdot \mathrm{kg}^{-1}$ of thermoneutral water, whereas, following the second period, they ingested $7.5 \mathrm{~g} \cdot \mathrm{kg}^{-1}$ of ice slurry. Both Tgint and Trec were measured continuously. The TPs were swallowed $10 \mathrm{~h}$ prior to the experiments. A bias $\leq 0.27^{\circ} \mathrm{C}$ was taken as an indication that Tgint and Trec provided similar core temperature indices. Mean biases and $95 \%$ limits of agreement during passive sitting, first exercise, water ingestion, second exercise, and ice slurry ingestion periods were $0.16 \pm 0.53,0.13 \pm 0.41,0.21 \pm 0.70,0.17 \pm 0.50$, and $0.18 \pm 0.66{ }^{\circ} \mathrm{C}$, respectively. The rates of decrease in Tgint and Trec did not differ between the water and ice slurry ingestion periods. Our results indicate that ice slurry ingestion following exercise does not impact TP-derived assessment of Tgint compared with Trec.
\end{abstract}

Keywords: core body temperature; exercise; heat stress; ice slurry ingestion; telemetry; temperature measurement

\section{Introduction}

The telemetric pill (TP) is being increasingly used by exercise scientists, physiologists, sports medicine physicians, and athletic trainers interested in determining and understanding the impact of various interventions on human thermoregulation during exercise [1]. The TP is typically swallowed several hours before data collection and therefore provides a measure of gastrointestinal temperature (Tgint). The popularity of this measurement technique is not surprising, since there are obvious advantages associated with its use. For instance, compared to the more traditional, valid, and accepted method to assess core temperature during exercise, i.e., through the rectum via the use of a rectal probe [2], it is non-invasive, associated with little burden for users, can be used relatively easily under field conditions, and is not subject to negative preconceptions [3,4].

It has generally been accepted that Tgint represents a reliable measurement of core temperature during exercise [3]. However, when the literature is closely scrutinized, one can observe that this is not always true, especially when cold fluids are being consumed. For example, Easton et al. [5] had 
participants ingest a TP $8 \mathrm{~h}$ prior to the commencement of the exercise trial and observed, on three occasions, Tgint below $30^{\circ} \mathrm{C}$ immediately following water $\left(5^{\circ} \mathrm{C}\right)$ ingestion. Similarly, Wilkinson et al. [6] observed that, $8 \mathrm{~h}$ following the ingestion of a TP, Tgint could still decrease by as much as $6{ }^{\circ} \mathrm{C}$ immediately following water $\left(5-8^{\circ} \mathrm{C}\right)$ intake. During a half-marathon, Savoie et al. [4] had runners consume $200 \mathrm{~mL}$ of water $\left(4^{\circ} \mathrm{C}\right)$ every $2 \mathrm{~km}$ and observed that, as the run progressed, the disagreement between Trec and Tgint slowly but steadily kept increasing, with a mean final disagreement between sites of $1.1{ }^{\circ} \mathrm{C}$. This was observed even though TPs were ingested $10 \mathrm{~h}$ before experiments started. Albeit in the former two studies, measurement errors in Tgint could be attributable to a direct contact of water with the TP, Savoie et al. [4] argued that, in their study, the creation of a heat exchanger surface may have been the culprit.

Gastrointestinal transit time may substantially vary within and between individuals [7]. Thus, following ingestion, the location of a TP within the gastrointestinal tract may be highly variable within and between individuals [8], and the closer a TP resides to a heat exchange medium at the upper gastrointestinal level, the higher the possibility that the temperature would be impacted. Ingestion of ice slurry has been demonstrated to decrease core temperature through the creation of a heat sink at the upper gastrointestinal level [9]. To this effect, Ihsan et al. [10] examined the effect of ice ingestion as a pre-cooling strategy on subsequent exercise performance. Participants ingested a TP $8-10 \mathrm{~h}$ prior to performance trials and consumed $6.8 \mathrm{~g} \cdot \mathrm{kg}^{-1}$ of either ice slurry or water $\left(27^{\circ} \mathrm{C}\right) 30 \mathrm{~min}$ before exercise. Water ingestion did not impact Tgint, but during ice slurry intake, Tgint decreased on average by $1.1^{\circ} \mathrm{C}$ and, according to Lee [11], at least one individual showed a decrease in Tgint below $35^{\circ} \mathrm{C}$. Similarly, Zimmerman et al. [12] had participants consume $7 \mathrm{~g} \cdot \mathrm{kg}^{-1}$ of either ice slurry or water (thermoneutral) over a $30 \mathrm{~min}$ period preceding exercise. Again, Tgint was not impacted by water ingestion, but during the ice slurry intake, Tgint decreased on average by $0.9^{\circ} \mathrm{C}$. Interestingly, studies have shown that the ingestion of $7.5 \mathrm{~g} \cdot \mathrm{kg}^{-1}$ of ice slurry does not decrease Trec by more than $0.6{ }^{\circ} \mathrm{C}[9,13-15]$. Based on this observation, it is tempting to propose the idea that ice slurry ingestion may have more impact on Tgint than Trec since the TP resides more closely to the heat sink than the rectal probe. However, we are aware of no studies that concurrently examine the kinetics of Tgint and Trec following ice slurry ingestion.

Given the extent of the use of TP in the assessment of Tgint, it is important to determine whether TP can reliably be used and provide good estimates of Tgint under situations where heat sinks are created at the upper gastrointestinal level. Therefore, the goal of this study was to examine the impact of an ice slurry-induced heat sink following an exercise-induced increase in core body temperature on the degree of agreement between Tgint and Trec. A greater impact of the heat sink on Tgint than Trec would be reflected by a faster rate of decline in Tgint than Trec and a lower absolute Tgint than Trec following ingestion.

\section{Materials and Methods}

\subsection{Participants}

Eleven healthy and physically active men $(32 \pm 7$ years; $1.77 \pm 0.6 \mathrm{~m} ; 74 \pm 6 \mathrm{~kg}$; body surface area: $1.91 \pm 0.08 \mathrm{~m}^{2}$; resting heart rate: $57 \pm 8$ beats $\mathrm{min}^{-1}$; and estimated maximal heart rate: $185 \pm 5$ beats $\cdot \mathrm{min}^{-1}$ ) participated in this study. Participants were explained the study protocol and its associated risks, after which written informed consent was obtained. The University of Sherbrooke Institutional Review Board approved all protocol procedures (\#2016-1144).

\subsection{Overview of the Study}

Participants came to the laboratory on two occasions (at 8:00 am for 9 participants and 04:30 pm for 2 participants). Baseline measurements were taken on the first visit, whereas during the second visit, participants underwent a research protocol where continuous measurements of Tgint and Trec were 
obtained during a sequence comprising one sitting period, a running or a cycling exercise followed by water ingestion, and another running or cycling exercise followed by ice slurry ingestion (Figure 1).

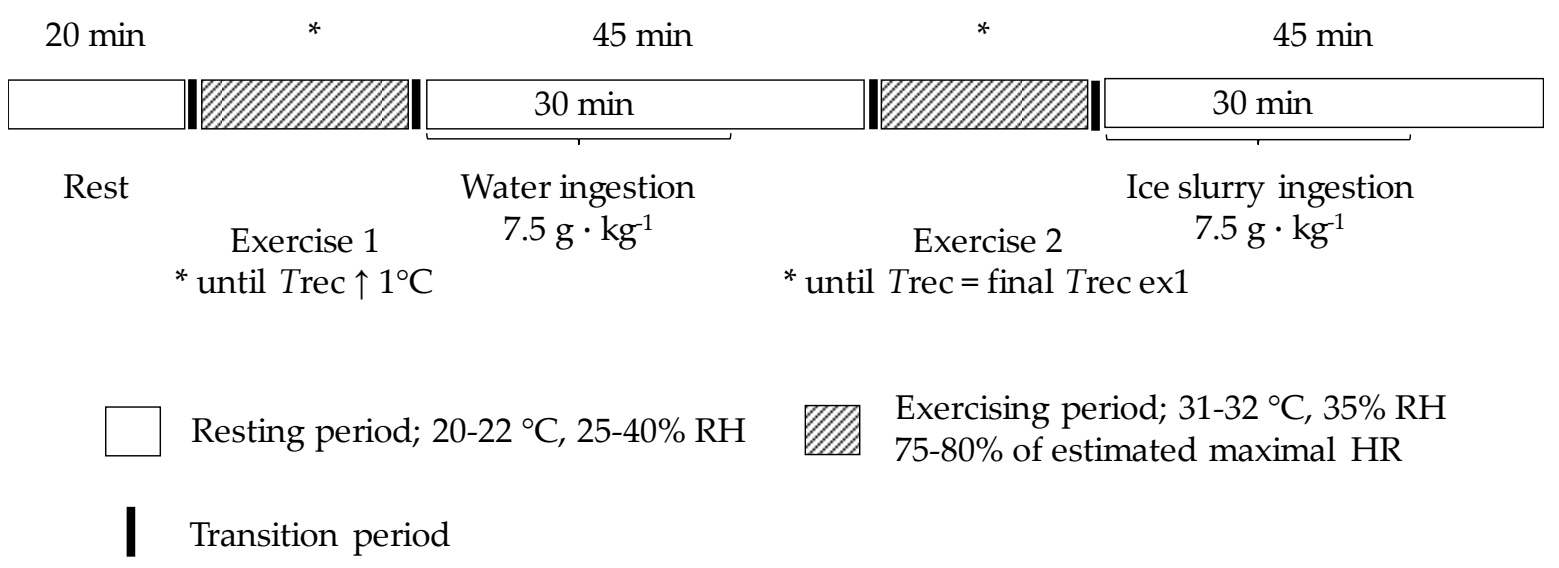

Figure 1. Schematic representation of the design of the study. HR: heart rate; Trec: rectal temperature; RH: relative humidity.

\subsection{Preliminary Visit}

Participants' height, body mass, resting heart rate, and blood pressure were measured during the preliminary visit. Post-void nude body mass and height were respectively measured using a digital scale (Bx-300+, Atron Systems, NJ, USA) and a wall stadiometer. After 3 min of seated rest, heart rate and blood pressure were taken with a digital sphygmomanometer (Welch Allyn 420 series, NY, USA). The equation developed by Tanaka et al. [16] was used to compute the estimated maximal heart rate.

\subsection{Pre-Experimental Protocol}

For the experiment, all participants were asked to report to the laboratory in a well-fed and rested state. Also, they were requested to consume $250 \mathrm{~mL}$ of water $1 \mathrm{~h}$ before their arrival and then to remain fasted. The TP (CorTemp HQ Inc., FL, USA) was swallowed $10 \mathrm{~h}$ prior to arriving at the laboratory for all the participants.

\subsection{Experimental Protocol}

Upon arrival at the laboratory, participants voided their bladder, provided a urine sample for urine specific gravity (USG) assessment, and their nude body mass was determined (Bx-300+, Atron Systems, NJ, USA). They then put on their running or cycling clothing and were instrumented with a chest electrode to measure heart rate, a rectal probe, and four epidermal probes affixed above the left pectoral muscle, the left forearm, the left thigh, and the left calf muscle. Then, the participants underwent the research protocol, which consisted of five phases that were performed sequentially: (1) a 20 min sitting period $\left(20-22{ }^{\circ} \mathrm{C}, 25-40 \%\right.$ relative humidity $\left.(\mathrm{RH})\right)$; (2) a running or a cycling exercise (75-80\% of estimated maximal heart rate [16]) conducted at $31-32{ }^{\circ} \mathrm{C}$ with $35 \% \mathrm{RH}$ aiming to increase Trec $1{ }^{\circ} \mathrm{C}$ above that measured at the end of the $20 \mathrm{~min}$ sitting period; (3) a $45 \mathrm{~min}$ sitting period while ingesting, over the first $30 \mathrm{~min}, 7.5 \mathrm{~g} \cdot \mathrm{kg}^{-1}$ of water (provided at mean Trec and Tgint) every $10 \mathrm{~min}$; (4) a second exercise period aiming to increase $\operatorname{Trec} 1{ }^{\circ} \mathrm{C}$ above that measured at the end of the $20 \mathrm{~min}$ sitting period; and (5) a 45 min sitting period while ingesting, over the first $30 \mathrm{~min}, 7.5 \mathrm{~g} \cdot \mathrm{kg}^{-1}$ of ice slurry $\left(-1{ }^{\circ} \mathrm{C}\right)$ every $10 \mathrm{~min}$. Participants could choose between a cycling or a running exercise, but the same mode of exercise as well as the same exercise intensity was used during the first and the second exercise bouts.

Transition times were not standardized between phases or participants but were performed as rapidly as possible. Participants could not consume fluids during the experiment other than the water and ice slurry provided during the sitting periods. However, they could rinse their mouths with cold 
water during the exercise periods. At the end of the experiment, participants voided their bladder, and a final nude body mass was taken.

\subsection{Measurements and Procedures}

Change in body mass from pre- to post-experiment period, corrected for water and ice slurry intakes and urine production, was used to estimate sweat loss. The dehydration level was estimated using the following formula:

Dehydration level: ( $\Delta$ pre-post experiment body mass/pre-experiment body mass) $\times 100$

Urine specific gravity was measured using a digital refractometer (PAL-10S, Atago, WA, USA). Rectal and gastrointestinal temperatures were respectively measured with a calibrated YSI 401 wired rectal probe (Yellow Springs Instrument, OH, USA) and a TP (CorTemp HQ Inc., FL, USA). The wired rectal probe was inserted $15 \mathrm{~cm}$ beyond the anal sphincter and securely held in place with the aid of a lightweight harness. The YSI probe was connected to a high precision digital thermometer (Traceable 4005, Control Company, TX, USA). Gastrointestinal temperature signals were recorded with a single CorTemp Data Recorder (HQ Inc, Palmetto, FL, USA) held in place at the participants' lower backs and base of the gluteal muscles. Both temperatures were recorded continuously every $20 \mathrm{~s}$. The YSI probe and each TP were calibrated before the beginning of the experiment. Calibration was performed at four different temperatures $\left(37,38,39\right.$, and $40^{\circ} \mathrm{C}$ ) in a heated bath (Precision 281, Thermo Scientific, MA, USA) using a high precision, partial immersion, non-mercury glass thermometer (Thermo Scientific Ertco, USA). A 4-point regression line [17] was used to predict Tgint and Trec. Skin temperature was measured with YSI 409 B probes (Yellow Springs Instrument, OH, USA) and computed according to Ramanathan [18]. The four probes were held in place using Transpore tape ( $3 \mathrm{M}, \mathrm{USA})$ and connected to a switch box linked to a high precision digital thermometer (Traceable 4005, Control Company, TX, USA). Throughout all five phases of the experiment, heart rate was measured with a T-31 Polar electrode linked to a Vantage NV Polar heart rate monitor (Polar USA, NY, USA). To produce the ice slurry, cubes of ice were shaved using a commercially available instrument (Hamilton Beach, USA). More precisely, the shaved ice was mixed with water $\left(4^{\circ} \mathrm{C}\right)$ in a $64: 36 \%$ ice-to-water ratio. Moreover, $\sim 22 \mathrm{~g}$ of glucose-fructose syrup (orange, grape, or raspberry) were added to the ice slurry in order to provide energy and increase palatability. Then, the ice slurry was conserved in the lower back of a refrigerator $\left(2-4{ }^{\circ} \mathrm{C}\right)$. Finally, a few steps were necessary to produce the water provided at mean Trec and Tgint. First, $\sim 2.5 \mathrm{~L}$ of water were kept at $40{ }^{\circ} \mathrm{C}$ in a heated bath. Once the time came to produce the solution, $\sim 5 \mathrm{~g} \cdot \mathrm{kg}^{-1}$ of this water were poured into an insulated bottle that contained a high precision digital thermometer. Then, rapidly but meticulously, $4{ }^{\circ} \mathrm{C}$ water was poured into the bottle until the desired temperature was obtained. Afterwards, the desired quantity of water, i.e., $2.5 \mathrm{~g} \cdot \mathrm{kg}^{-1}$, was transferred to another insulated bottle.

\subsection{Statistical Analyses}

Normality of data distribution was tested with the Shapiro-Wilk test. Acceptable agreement between measurement techniques was taken as a bias $\leq 0.27^{\circ} \mathrm{C}$ [2]. Heart rate and mean skin temperature data were analysed using one-way repeated measures analysis of variance (ANOVA). Greenhouse-Geisser corrections were applied when sphericity was violated. Paired sample $t$-tests or Wilcoxon signed-rank tests were used to determine whether biases were systematic. A two-way repeated measures ANOVA was used to determine the effect of water and ice slurry conditions on Tgint vs. Trec. Relative validity was assessed with the Pearson product-moment correlation coefficient (r). Absolute validity (random error statistics) was determined with the computation of the typical error of measurement (TEM), coefficient of variation (CV), and the Bland-Altman 95\% limits of agreement (LoA). Heteroscedasticity was assessed using a linear regression model with Trec-Tgint as the dependent variable and Trec-Tgint/2 as the independent variable. Significant heteroscedasticity 
was observed for phases 3 to 5 and data comprising the entire experiment. However, no correction was performed given the weak relationship for each comparison. Given the different exercise times within and between participants, results for the exercise phases are reported based on percentage of maximal exercise time. Statistics were performed with the 2018 Microsoft Office Excel (Microsoft, WA, USA) and IBM SPSS Statistics (version 25, NY, USA) software. Results were considered significant at $p \leq 0.05$. Data are reported as means \pm SD unless stated otherwise.

\section{Results}

\subsection{Temperature and Relative Humidity}

The average temperature and the RH inside the heat chamber were respectively $31.7 \pm 1.3^{\circ} \mathrm{C}$ and $33.1 \pm 5.5 \%$ for the first exercise period and $30.8 \pm 1.1^{\circ} \mathrm{C}(p=0.10)$ and $33.5 \pm 5.3 \%(p=0.71)$ for the second exercise period. Sitting periods were performed at an ambient temperature of $20-22{ }^{\circ} \mathrm{C}$ with $25-40 \%$ RH.

\subsection{Fluid Balance}

Participants were well hydrated before the start of the experiment, as supported by a mean USG of $1.016 \pm 0.009 \mathrm{~g} \cdot \mathrm{mL}^{-1}$. Mean USG increased to $1.021 \pm 0.005 \mathrm{~g} \cdot \mathrm{mL}^{-1}$ at the end of the experiment $(p=0.01)$. Average ice slurry and water intake was $555 \pm 41 \mathrm{~g}$. Following exercise, urine production, sweat loss, sweat rate, and dehydration level amounted to $256 \pm 122 \mathrm{~mL}, 1593 \pm 441 \mathrm{~mL}, 540 \pm 96 \mathrm{~mL} \cdot \mathrm{h}^{-1}$ and $1.0 \pm 0.7 \%$ of body mass, respectively.

\subsection{Exercise Time, Heart Rate, and Exercise Intensity}

The mean exercise times for the first and the second exercise periods were $24.8 \pm 9.5$ and $25.0 \pm 13.6 \mathrm{~min}(p=0.72)$, respectively. Figure 2 shows the changes in heart rate across time for all five phases. Average heart rate during the first $\left(152 \pm 12\right.$ beats $\left.\cdot \mathrm{min}^{-1}\right)$ and the second $\left(153 \pm 15\right.$ beats $\left.\cdot \mathrm{min}^{-1}\right)$ exercise periods corresponded respectively to $78 \pm 5$ and $78 \pm 6 \%$ of estimated maximal heart rate $(p=0.53)$. Except for the $20 \mathrm{~min}$ passive sitting period $(p=0.18)$, a significant time effect was observed for the changes in mean heart rate across time for all other phases $(p<0.01)$.

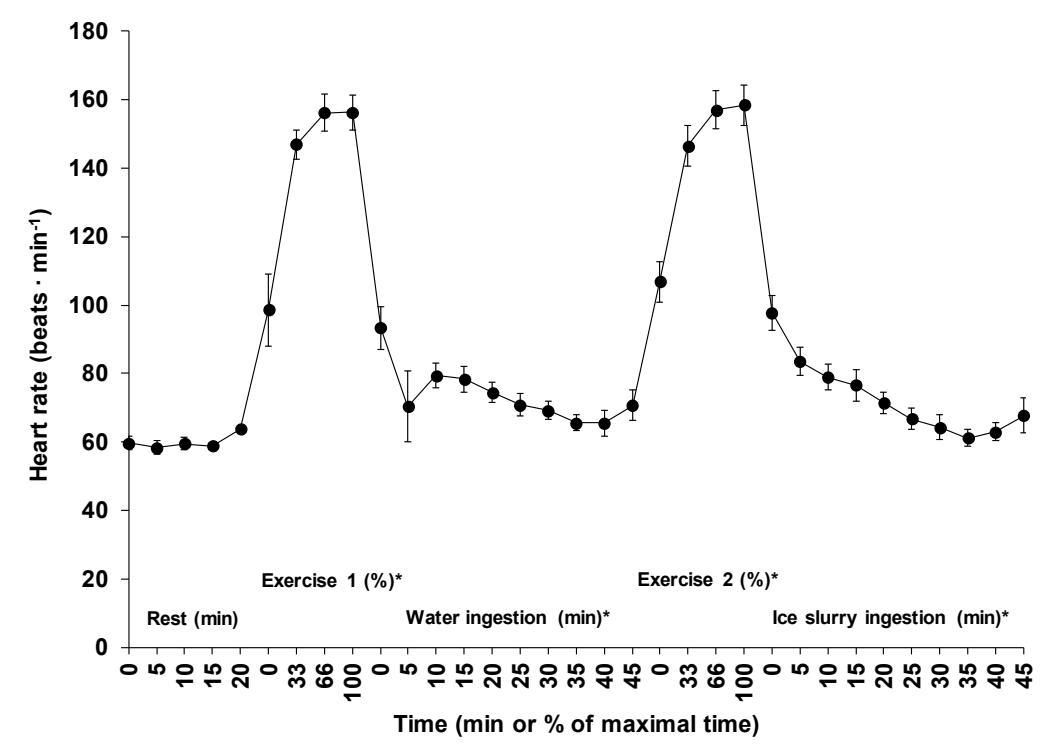

Figure 2. Changes in heart rate across time for all five phases of the experiment. Results are means \pm SD.

*: significant time effect throughout the phase. 


\subsection{Mean Skin Temperature}

Figure 3 illustrates the changes in mean skin temperature across time for all five phases. Average skin temperature during passive sitting, first exercise, water ingestion, second exercise, and ice slurry ingestion periods were $31.7 \pm 0.7,34.3 \pm 1.2,32.4 \pm 0.8,34.2 \pm 0.9$, and $32.2 \pm 0.9{ }^{\circ} \mathrm{C}$, respectively. Except for the 20 min passive sitting period ( $p=0.27$ ), a significant time effect was observed for the changes in mean skin temperature across time for all other phases. The average mean skin temperature increased by $1.7 \pm 1.1$ and $2.2 \pm 0.9^{\circ} \mathrm{C}$ during the first and the second exercise periods, respectively $(p<0.01)$ and decreased by $2.3 \pm 1.6$ and $2.8 \pm 1.2^{\circ} \mathrm{C}$, respectively, during the water and the ice slurry ingestion periods $(p<0.01)$.

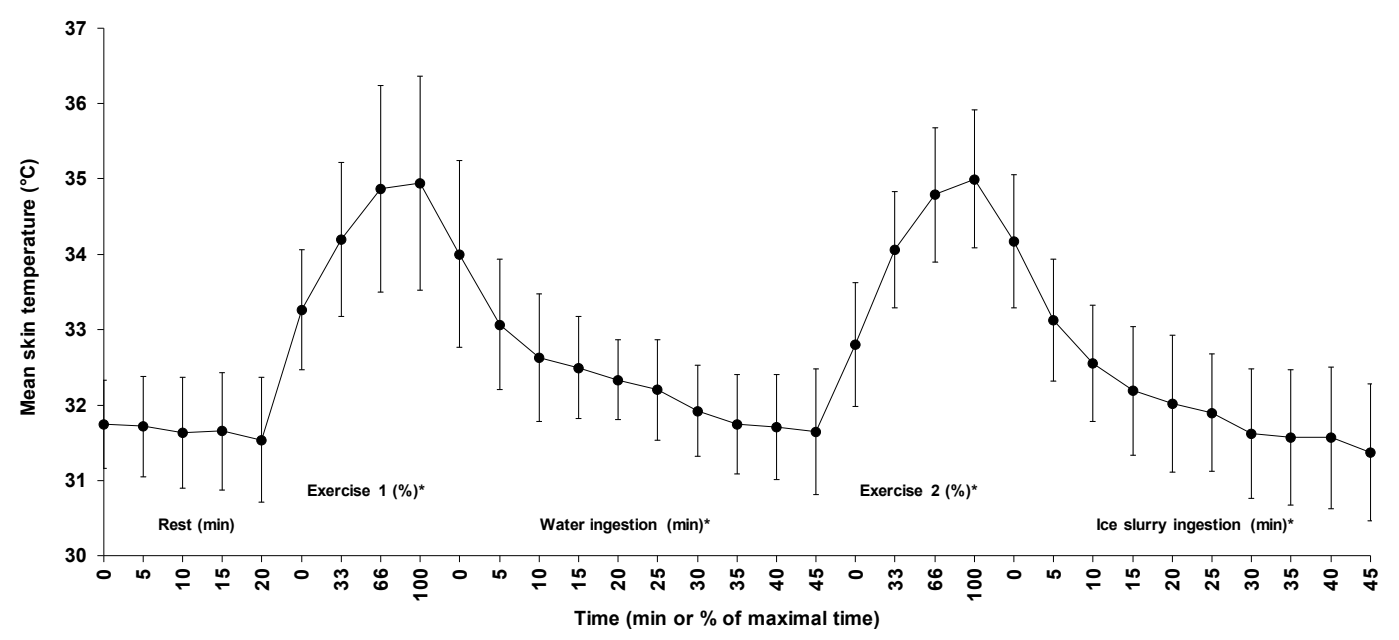

Figure 3. Changes in mean skin temperature for all five phases of the experiment. Results are means \pm SD. *: significant time effect throughout the phase.

\subsection{Heat Sink}

The heat sink was successfully created, as evidenced by a steeper decrease in both Tgint and Trec during the ice slurry than the water ingestion period (Figure 4). On average, during the water ingestion period, Tgint decreased by $0.82 \pm 0.35$, whereas Trec decreased by $0.79 \pm 0.16{ }^{\circ} \mathrm{C}$. On the other hand, during the ice slurry ingestion period, Tgint decreased by $1.24 \pm 0.27$, whereas Trec decreased by $1.15 \pm 0.24^{\circ} \mathrm{C}$. Altogether, ice slurry ingestion further decreased Tgint by $0.41 \pm 0.40$ compared with $0.35 \pm 0.21{ }^{\circ} \mathrm{C}$ for Trec $(p=0.72)$. The rates of decrease of both Tgint and Trec were faster $(p<0.01)$ in the ice slurry than the water condition.

\subsection{Gastrointestinal and Rectal Temperatures}

Figure 4 presents the changes in Tgint and Trec across time during the five phases. Mean Tgint and Trec during the entire experiment were respectively of the order of $38.0 \pm 0.4$ and $37.8 \pm 0.5^{\circ} \mathrm{C}$ $(p<0.01)$. With the exception of Tgint during the passive sitting period, a significant time effect was observed across all five phases with, as expected, a decline in mean Tgint and Trec during the water and the ice slurry ingestion periods, with the opposite occurring during both exercise periods (all $p<0.01$ ). The rates of change in Tgint and Trec across all five phases were similar with values of $-0.002 \pm 0.008$ vs. $-0.005 \pm 0.006(p=0.50), 0.047 \pm 0.020$ vs. $0.047 \pm 0.017(p=0.92),-0.018 \pm 0.008$ vs. $-0.018 \pm 0.004$ $(p=0.17), 0.041 \pm 0.022$ vs. $0.036 \pm 0.012(p=0.74)$, and $-0.028 \pm 0.006$ vs. $-0.026 \pm 0.005^{\circ} \mathrm{C} \cdot \mathrm{min}^{-1}$ $(p=0.47)$ for phases $1,2,3,4$, and 5 , respectively. 


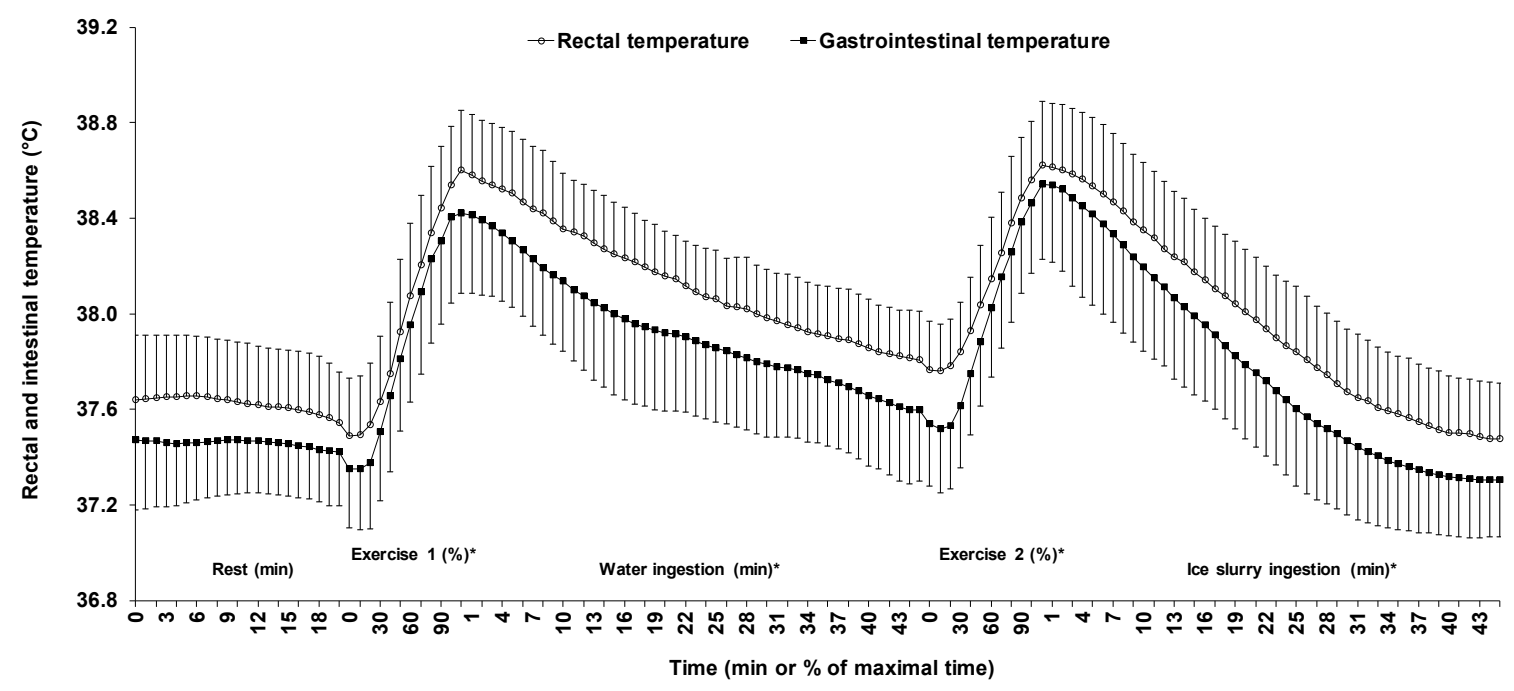

Figure 4. Changes in gastrointestinal and rectal temperatures across time for all five phases of the experiment. Results are means \pm SD. *: significant time effect throughout the phase.

Data related to the relative and the absolute comparisons between Tgint and Trec are reported in Table 1. For all five phases, Trec was greater than Tgint (all $p<0.01$ ), with all biases being systematic, including the global bias $(p<0.01)$. In none of the five phases was the mean bias between Trec and Tgint greater than the a priori established threshold of $\leq 0.27^{\circ} \mathrm{C}$. There was a pattern where the agreement between Trec and Tgint was stronger during the exercise than the resting periods. In fact, biases, CVs, TEMs, and 95\% LoAs were lower during the former than the latter periods, with little variation between the water and the ice slurry ingestion periods. Pearson product-moment correlation coefficients followed the same pattern and were higher during the exercise than the resting periods. However, the relationship between Trec and Tgint was stronger with ice slurry than water ingestion.

Table 1. Comparisons between gastrointestinal and rectal temperatures.

\begin{tabular}{cccccc}
\hline Periods & Bias $\left({ }^{\circ} \mathbf{C}\right)[\mathbf{9 5 \%} \mathbf{C I}]$ & TEM $\left({ }^{\circ} \mathbf{C}\right)$ & CV (\%) & 95\% LoA $\left({ }^{\circ} \mathbf{C}\right)$ & $r$ \\
\hline Passive sitting & $0.16[0.13-0.20]^{*}$ & \pm 0.27 & \pm 0.40 & \pm 0.53 & 0.33 \\
Exercise 1 & $0.13[0.09-0.16]^{*}$ & \pm 0.21 & \pm 0.33 & \pm 0.41 & 0.91 \\
Water ingestion & $0.21[0.18-0.24]^{*}$ & \pm 0.36 & \pm 0.58 & \pm 0.70 & 0.48 \\
Exercise 2 & $0.17[0.12-0.21]^{*}$ & \pm 0.25 & \pm 0.40 & \pm 0.50 & 0.81 \\
Ice slurry ingestion & $0.18[0.15-0.21]^{*}$ & \pm 0.34 & \pm 0.60 & \pm 0.66 & 0.77 \\
\hline Mean & $0.18[0.17-0.20]^{*}$ & \pm 0.32 & \pm 0.52 & \pm 0.63 & 0.73 \\
\hline
\end{tabular}

Bias: rectal-intestinal temperature; CI: confidence interval; CV: coefficient of variation; LoA: $95 \%$ limits of agreement; $r$ : Pearson product-moment correlation coefficient; TEM: typical error of measurement; *: systematic bias.

\section{Discussion}

The aim of this study was to determine whether TP-derived Tgint and rectal probe-derived Trec are impacted differently by the creation of an ice slurry-induced heat sink following exercise.

Participants were requested to complete two bouts of exercise, after which, on one occasion, thermoneutral water was consumed and, on the other, ice slurry was consumed. The goal of the water trial was to characterize the pattern of decline in Tgint and Trec while considering the effect of fluid ingestion on thermal and non-thermal effectors of body heat loss [19]. Gastrointestinal and rectal temperature responses were examined after rather than during exercise to reduce the potential confounding effect of (1) gastric emptying on the rate of fluid transfer to the intestines [20]; (2) thermal inertia, which may have resulted in different amounts of body heat storage among conditions [21]; and (3) movement-induced gastric motility. The differences between Trec and Tgint during the thermoneutral water and the ice slurry ingestion periods were similar and $\leq 0.27^{\circ} \mathrm{C}$, our a priori 
established threshold for non-difference between sites. From a practical standpoint, these results indicate that, following exercise, the monitoring of Tgint is unlikely to be impacted by a cooling of the upper gastrointestinal tract induced by a mass of ice slurry of about $555 \mathrm{~g} \cdot 74 \mathrm{~kg}$ body mass ${ }^{-1}$.

Rectal temperature was systematically higher than Tgint throughout the different phases of the experiment. Previously published studies have reported conflicting findings regarding the direction of the bias between Trec and Tgint during multi-phase experiments comprising resting and exercising periods with or without fluid ingestion [1]. For instance, Casa et al. [2] $\left(0.19{ }^{\circ} \mathrm{C}\right)$, Kolka et al. [22] $\left(0.18{ }^{\circ} \mathrm{C}\right)$, and Gosselin et al. [23] $\left(0.20^{\circ} \mathrm{C}\right)$ reported higher Trec than Tgint. On the other hand, Gant et al. [24] $\left(0.15^{\circ} \mathrm{C}\right)$ and Teunissen et al. [25] $\left(0.14^{\circ} \mathrm{C}\right)$ observed Tgint to be higher than Trec. Our findings add to the current literature and indicate that an acceptable difference between Trec and Tgint during experiments composed of various passive and active phases with or without fluid or ice slurry ingestion should lie within $\pm 0.20^{\circ} \mathrm{C}$, which is reasonable from a technical and a physiological point of view given that the daily variability in $T$ rec is $\sim \pm 0.25{ }^{\circ} \mathrm{C}$ [26].

Although the overall mean bias $\left(0.18^{\circ} \mathrm{C}\right)$ between Trec and Tgint was under what we consider to be an acceptable difference between sites, i.e., $<0.27^{\circ} \mathrm{C}$, the mean $95 \% \mathrm{LoA}\left( \pm 0.63^{\circ} \mathrm{C}\right)$ indicates that there may be wide variations between sites within and between individuals. Given that the bias was in favor of a greater Trec than Tgint over the entire research protocol, as illustrated in Figure 4, this may have an important implication for the practitioners or the coaches using TPs to monitor core temperature. Indeed, in the case of a suspected exertional heat stroke, our findings indicate that Trec should also be taken instead of relying solely on Tgint in order to establish the correct diagnostic and initiate the proper treatment.

Whether in absolute or relative terms, the patterns of temporal changes in temperature between Trec than Tgint during the water and the ice slurry ingestion periods were similar, thereby implying that ice slurry ingestion did not impact Tgint differently than Trec. Several reasons may be thought of to explain this observation. The amount of cooling in the face of hyperthermia may not have been important enough such that thermal equilibrium with the surrounding tissues was attained prior to reaching the TPs. The volume of warm blood returning to the upper gastrointestinal area, and hence convective heat flow, may have been more important than in the rectal area, therefore hiding any potential effect of the heat sink on Tgint. Moreover, a higher metabolic rate (driven by fluid absorption) of the upper intestinal region than in the rectum would have acted to increase local temperature and therefore mask the effect of the heat sink. However, the two preceding scenarios are improbable, as this would have been reflected during the water ingestion period as a lower rate of decline in Tgint than Trec, which was not the case. A greater thermal inertia of the TP is unlikely to have been responsible for the observed findings. In fact, Gosselin et al. [23] demonstrated that, when inserted concurrently in the rectum, the absolute and the relative changes in temperature between a TP and wired rectal probe are similar.

An interesting observation of this study is that the divergence in temperatures between Trec and Tgint was more important during the ingestion periods than during the exercise periods, as underlined by higher TEMs, CVs, and 95\% LoAs during the former than the latter period. During exercise, the sympathetic activity-driven arteriole vasoconstriction was likely similar between the rectal and the upper gastrointestinal area, thereby producing a more uniform response between regions and among individuals due to a greater reliance on conduction than convection for heat exchange. On the other hand, following exercise, heat exchange with surrounding tissues became more reliant upon convection, and the rate as well as the total amount of blood perfusing the rectal and the upper gastrointestinal area likely differed among individuals, explaining the greater variability in results.

The present findings need to be interpreted with the following limitations in mind. First, we provided a mass of ice slurry of $7.5 \mathrm{~g} \cdot \mathrm{kg}^{-1}$, which created an estimated heat debt of $210 \mathrm{~kJ}$. It is not impossible that a higher mass of ice slurry would have unraveled a difference between Trec and Tgint. However, from a practical perspective, consumption of a higher mass of ice slurry would have been difficult to sustain by participants within the $30 \mathrm{~min}$ timeframe allowed for ingestion. Moreover, 
the amount of ice slurry administered is in line with the mass most studies examining the impact of ice slurry ingestion on thermoregulation have provided, which enhances the external validity of our findings. Second, the exact location of the TP within the gastrointestinal tract was unknown at the time of the water and the ice slurry ingestion periods. Since the TPs were ingested $10 \mathrm{~h}$ prior to starting the experiments, it is therefore reasonable from a physiological standpoint to believe that they may have been at a distance too far from the heat exchange surface to be impacted differently than Trec. However, timing of ingestion of the TPs was deliberately chosen to optimize the external and the ecological validity of findings, as it has been suggested that it is the optimal timeframe for preventing external contamination from fluid or food consumption [4]. Finally, the created heat sink could exchange energy with all tissues surrounding its center. However, as the TPs were located beneath the center of the heat sink, only a certain proportion of tissues impacted by the heat sink could exchange heat with the sensors.

In conclusion, the present results indicate that the ingestion of ice slurry following exercise does not impact measurement of Tgint compared with Trec, given that the ingestion of the TP occurs $10 \mathrm{~h}$ prior. These results have implications for athletic trainers, sports medicine physicians, scientists, physiologists, and military personnel who use TPs to monitor core temperature changes following exercise using a variety of cooling procedures, including ice slurry ingestion at a mass of $7.5 \mathrm{~g} \cdot \mathrm{kg}$ body mass $^{-1}$.

Author Contributions: Conceptualization, A.D.L.F. and E.D.B.G.; Formal analysis, T.A.D. and E.D.B.G.; Investigation, A.D.L.F., J.G., J.B. and E.D.B.G.; Methodology, A.D.L.F. and E.D.B.G.; Resources, E.D.B.G.; Supervision, E.D.B.G.; Validation, E.D.B.G.; Writing—original draft, T.A.D., J.G. and E.D.B.G.; Writing—review \& editing, T.A.D., A.D.L.F., J.G., D.J. and E.D.B.G.

Funding: This research was funded by a grant provided by the Université de Sherbrooke.

Acknowledgments: The authors thank all participants who participated in this study. This study was made possible through a research grant provided by the Université de Sherbrooke.

Conflicts of Interest: The authors report no conflicts of interest associated with this manuscript.

\section{References}

1. Byrne, C.; Lim, C.L. The ingestible telemetric body core temperature sensor: A review of validity and exercise applications. Br. J. Sports Med. 2007, 41, 126-133. [CrossRef]

2. Casa, D.J.; Becker, S.M.; Ganio, M.S.; Brown, C.M.; Yeargin, S.W.; Roti, M.W.; Siegler, J.; Blowers, J.A.; Glaviano, N.R.; Huggins, R.A.; et al. Validity of Devices That Assess Body Temperature During Outdoor Exercise in the Heat. J. Athl. Train. 2007, 42, 333-342. [PubMed]

3. Bongers, C.C.; Hopman, M.T.; Eijsvogels, T.M. Using an Ingestible Telemetric Temperature Pill to Assess Gastrointestinal Temperature During Exercise. J. Vis. Exp. 2015, 104, e53258. [CrossRef] [PubMed]

4. Savoie, F.A.; Dion, T.; Asselin, A.; Gariepy, C.; Boucher, P.M.; Berrigan, F.; Goulet, E.D.B. Intestinal temperature does not reflect rectal temperature during prolonged, intense running with cold fluid ingestion. Physiol. Meas. 2015, 36, 259-272. [CrossRef] [PubMed]

5. Easton, C.; Fudge, B.W.; Pitsiladis, Y.P. Rectal, telemetry pill and tympanic membrane thermometry during exercise heat stress. J. Therm. Boil. 2007, 32, 78-86. [CrossRef]

6. Wilkinson, D.M.; Carter, J.M.; Richmond, V.L.; Blacker, S.D.; Rayson, M.P. The Effect of Cool Water Ingestion on Gastrointestinal Pill Temperature. Med. Sci. Sports Exerc. 2008, 40, 523-528. [CrossRef]

7. Birkebaek, N.H.; Memmert, K.; Mortensen, J.; Dirksen, H.; Christensen, M.F. Fractional gastrointestinal transit time: Intra- and interindividual variation. Nucl. Med. Commun. 1990, 11, 247-252. [CrossRef] [PubMed]

8. McKenzie, J.; Osgood, D. Validation of a new telemetric core temperature monitor. J. Therm. Boil. 2004, 29, 605-611. [CrossRef]

9. Siegel, R.; Maté, J.; Brearley, M.B.; Watson, G.; Nosaka, K.; Laursen, P.B. Ice Slurry Ingestion Increases Core Temperature Capacity and Running Time in the Heat. Med. Sci. Sports Exerc. 2010, 42, 717-725. [CrossRef]

10. Ihsan, M.; Landers, G.; Brearley, M.; Peeling, P. Beneficial effects of ice ingestion as a precooling strategy on 40-km cycling time-trial performance. Int. J. Sports Physiol. Perform. 2010, 5, 140-151. [CrossRef] 
11. Lee, J.K.W. Erroneous readings from ingestible temperature capsules due to ingestion of crushed ice. Int. J. Sports Physiol. Perform. 2011, 6, 5-6.

12. Zimmermann, M.; Landers, G.; Wallman, K.E.; Saldaris, J. The Effects of Crushed Ice Ingestion Prior to Steady State Exercise in the Heat. Int. J. Sport Nutr. Exerc. Metab. 2017, 27, 220-227. [CrossRef]

13. Stevens, C.J.; Thoseby, B.; Sculley, D.V.; Callister, R.; Taylor, L.; Dascombe, B.J. Running performance and thermal sensation in the heat are improved with menthol mouth rinse but not ice slurry ingestion. Scand. J. Med. Sci. Sports 2016, 26, 1209-1216. [CrossRef]

14. Aldous, J.W.F.; Chrismas, B.C.R.; Akubat, I.; Stringer, C.A.; Abt, G.; Taylor, L. Mixed-methods pre-match cooling improves simulated soccer performance in the heat. Eur. J. Sport Sci. 2019, 19, 156-165. [CrossRef]

15. Siegel, R.; Maté, J.; Watson, G.; Nosaka, K.; Laursen, P.B. Pre-cooling with ice slurry ingestion leads to similar run times to exhaustion in the heat as cold water immersion. J. Sports Sci. 2012, 30, 155-165. [CrossRef]

16. Tanaka, H.; Monahan, K.D.; Seals, D.R. Age-predicted maximal heart rate revisited. J. Am. Coll. Cardiol. 2001, 37, 153-156. [CrossRef]

17. Challis, G.G.; Kolb, J.C. Agreement Between an Ingestible Telemetric Sensor System and a Mercury Thermometer Before and After Linear Regression Correction. Clin. J. Sport Med. 2010, 20, 53-57. [CrossRef]

18. Ramanathan, N.L. A new weighting system for mean surface temperature of the human body. J. Appl. Physiol. 1964, 19, 531-533. [CrossRef]

19. Mekjavic, I.B.; Eiken, O. Contribution of thermal and nonthermal factors to the regulation of body temperature in humans. J. Appl. Physiol. 2006, 100, 2065-2072. [CrossRef]

20. Costill, D.L.; Saltin, B. Factors limiting gastric emptying during rest and exercise. J. Appl. Physiol. 1974, 37, 679-683. [CrossRef]

21. Kenny, G.P.; Dorman, L.E.; Webb, P.; Ducharme, M.B.; Gagnon, D.; Reardon, F.D.; Hardcastle, S.G.; Jay, O. Heat Balance and Cumulative Heat Storage during Intermittent Bouts of Exercise. Med. Sci. Sports Exerc. 2009, 41, 588-596. [CrossRef]

22. Kolka, M.A.; Quigley, M.D.; Blanchard, L.A.; Toyota, D.A.; Stephenson, L.A. Validation of a temperature telemetry system during moderate and strenuous exercise. J. Therm. Boil. 1993, 18, 203-210. [CrossRef]

23. Gosselin, J.; Béliveau, J.; Hamel, M.; Casa, D.; Hosokawa, Y.; Morais, J.A.; Goulet, E.D. Wireless measurement of rectal temperature during exercise: Comparing an ingestible thermometric telemetric pill used as a suppository against a conventional rectal probe. J. Therm. Boil. 2019, 83, 112-118. [CrossRef]

24. Gant, N.; Atkinson, G.; Williams, C. The Validity and Reliability of Intestinal Temperature during Intermittent Running. Med. Sci. Sports Exerc. 2006, 38, 1926-1931. [CrossRef]

25. Teunissen, L.P.J.; De Haan, A.; De Koning, J.J.; Daanen, H.A.M. Telemetry pill versus rectal and esophageal temperature during extreme rates of exercise-induced core temperature change. Physiol. Meas. 2012, 33, 915-924. [CrossRef]

26. Goodman, D.A.; Kenefick, R.W.; Cadarette, B.S.; Cheuvront, S.N. Influence of Sensor Ingestion Timing on Consistency of Temperature Measures. Med. Sci. Sports Exerc. 2009, 41, 597-602. [CrossRef]

(C) 2019 by the authors. Licensee MDPI, Basel, Switzerland. This article is an open access article distributed under the terms and conditions of the Creative Commons Attribution (CC BY) license (http://creativecommons.org/licenses/by/4.0/). 